\title{
Determination of nicotine and cotinine in human blood by dried blood spot-LC-Orbitrap MS technique
}

\begin{abstract}
Background: Dried blood spot (DBS) have been applied in many application as alternate analytical solution for limitations and challenges in conventional methods, and validated methods demand by DBS technique are still required in nicotine (Nic) research area.

Aim: The development and validation of a new bioanalytical method for simultaneous determination of Nic and cotinine (Cot) in human blood using DBS and LC-Orbitrap MS technique.

Methods: The DBS was punched at $6.35 \mathrm{~mm}$ diameter and extracted by $10 \% \mathrm{w} / \mathrm{v}$ of trichloroacetic acid solution, containing Nic-d3 as an internal standard (IS). The extracted samples were then injected into a Kinetex-C18 column and eluted by mobile phase of methanol:water:formic acid (10:90:0.001, v/v/v). Chromatographic effect for DBS was investigated by decentralized disk punching (peripheral area). The developed method was validated according to European and American guidelines for bioanalytical method validation.

Results: Nic, Cot and IS were detected accurately by Orbitrap-MS using heated-ESI source at positive ions $\mathrm{m} / \mathrm{z}$ of $163.1235,177.1028$ and 166.1423 , respectively. The established calibration ranges for Nic and Cot were linear between $5-250 \mathrm{ng} / \mathrm{mL}$ and $10-500 \mathrm{ng} / \mathrm{mL}$, respectively. Within- and between-run measurements accuracy for Nic and Cot were all higher than $80 \%$ for LLOQ and $85 \%$ for QC levels, and the measurements precision for Nic and Cot were all within $15 \%$.

Conclusion: The developed method for Nic and Cot determination in human blood was successfully validated using DBS LC-Orbitrap MS technique. Nic and Cot investigation was not affected by DBS's chromatographic influence.
\end{abstract}

Keywords: dried blood spot, quantitation, lc-orbitrap ms, nicotine, cotinine
Volume 3 Issue 5 - 2016

\author{
Ahmad Abu Awwad, Oliver J Schmitz, Tawfiq \\ Arafat \\ 'University of Duisburg-Essen, Germany \\ 2University of Petra,Amman, Jordan
}

Correspondence: Oliver J Schmitz, Applied Analytical Chemistry, University of Duisburg-Essen Universitaetsstr, 545 I4I Essen, Germany, Tel +49 20I I833950, Fax +49 201183395I,Email olivers.schmitz@uni-due.de

Received: October 28, 2016 | Published: December 09, 2016
Abbreviations: NIC, nicotine; COT, cotinine; DBS, dried blood spot; IS, internal standard; TCA, trichloroacetic; TRC, torento research chemicals; DMPK, drug metabolism and pharmacokinetics; H-ESI, heated-electrospray ionization source; LLOQ, lower limit of quantification; QC, quality control; EMA, european medicines agency; MF, matrix factor; TIC, total ion current; RSD, relative standard deviation

\section{Introduction}

Nicotine Nic (Figure 1) has long been investigated in human body due to its adverse health consequence and addiction profile. ${ }^{1}$ It has also been used as insecticide due to its poisoning capabilities. ${ }^{2} \mathrm{Nic}$ enters bloodstream immediately through smoking by lung absorption , 3 and metabolized in liver mainly by CYP 2A6 to cotinine Cot (Figure 2) and other metabolites. For Nic determination in human blood, a traditional sampling procedure of drawing blood by venous cannula or syringe followed by multiple processing steps is usually used, but many restrictions of hospitalization demands, ethical agreements, and high costs are making challanges in Nic research area from human blood.

Dried blood spot (DBS) have been used technique for screening of metabolic disorders in newborns for decades, and have already became a popular sampling method for the quantitation of small molecules in blood. ${ }^{4}$ Using DBS technique has increased to manage high population-based studies, especially those for large-scale human bio monitoring, as well as studies involving children or small animals, since the skin-stick procedure is technically simple and doesn't need extensive training or expensive equipment, only a tiny volume of blood is enough, and handling blood specimens is relatively nonhazardous against pathogens risk, such as HIV. This technique has more recently been applied to drug metabolism, ${ }^{5}$ pharmacokinetic, ${ }^{6}$ therapeutic drug monitoring ${ }^{7}$ and toxicokinetic ${ }^{8}$ studies. DBS has applied in tobacco smoke research field as well, to provide an alternative sampling method for Cot determination in human blood as a biomarker for Nic intake in metabolism studies, ${ }^{9}$ secondhand smokers ${ }^{10}$ and newborn screening. ${ }^{11}$ Recently, Tretzel et al., ${ }^{12}$ has described an excellent application for DBS by automated samples extraction system, for determination of doping relevant compounds, using Nic and its major metabolites as an example for drugs testing in sport applications, ${ }^{12}$ which has achieved by multiple extraction steps (based on solid phase extraction), relatively low extraction recovery (25-40\%) and $10 \mathrm{~min}$ run time.

The current study demonstrates a validated bioanalytical method for simultaneous determination of Nic with Cot in human blood based on DBS technique, following a single extraction-reconstitution step. Subsequently, the validated method was then successfully applied to measure Nic and Cot levels in 12 healthy and smoker volunteers, and compared their DBS measurements with corresponding measurements from whole plasma (traditional blood sampling procedure), using LCOrbitrap MS technique as a highly accurate and sensitive detection 
system, to satisfy the sensitivity demand of small sample volume, where the current described method has the advantages of low running costs, single extraction step through direct reconstitution by trichloroacetic acid solution (TCA), short run time $(2.5 \mathrm{~min})$ and good extraction recovery from DBS. Furthermore, we investigated the influence of chromatographic effect for first time in Nic analysis by DBS technique.

\section{Experimental}

\section{Chemicals, reagents and materials}

Nic (purity 98\%) and deuterated Nic-d3 (purity 98\%) were obtained from Toronto research chemical (TRC Inc., Canada). Cot (purity 98\%) was obtained from Fischer Inc., Germany. Blood blank samples were harvested in heparinized tubes from healthy and nonsmoker donor. Deionized water, methanol and formic acid are in LC/MS-quality and purchased from Sigma Aldrich (Germany). FTA DMPK- filter cards, (Whatman ${ }^{\mathrm{TM}}$ 903, US-FDA approved type for quantitative analysis, ${ }^{13}$ ) were obtained from GE Healthcare.

\section{Instrumentation}

The LC-MS system was based on the benchtop Orbitrap-MS (Exactive, Thermo Fisher Scientific, Bremen-Germany), equipped with the heated-electrospray ionization (H-ESI) source and protected by a waste/detector Rheodyne divert valve, attached to (Accela ${ }^{\mathrm{TM}}$ ) LC system from Thermo Fisher Scientific (1250 Pump model) and controlled by Xcalibur 3.0.63 software for data management system.

\section{HPLC conditions}

An isocratic elution with methanol:water:formic acid $(10: 90: 0.001, \mathrm{v} / \mathrm{v} / \mathrm{v})$, delivered at constant flow rate of $0.4 \mathrm{~mL} / \mathrm{min}$ through an analytical Kinetex-C18column $(150 \times 2.1 \mathrm{~mm}, 5 \mu \mathrm{m})$ from Phenomenex ${ }^{\mathrm{TM}}$ (Germany) was used. The auto sampler tray temperature was set at $5^{\circ} \mathrm{C}$ and the injection volume was $20 \mu \mathrm{L}$.

\section{Mass spectrometric conditions}

The H-ESI source was operated in positive scan mode. The adjusted ion source parameters of nitrogen gas for sheath, auxiliary and sweep flow were at 45, 20,5 unit, respectively. Ion transfer capillary temperature and auxiliary gas heating temperature was at $300^{\circ} \mathrm{C}$ and $400^{\circ} \mathrm{C}$, respectively. The ion spray voltage was set at 4500 V. MS resolution option was set at high-50000@2Hz with a mass window of $0.1 \mathrm{D}$ for each analyt (Nic, Cot and Nic-d3).

\section{Standard solutions, calibration curve and QC samples}

Stock solutions of $1.0 \mathrm{mg} / \mathrm{mL}$ for Nic, Cot and Nic-d3 were prepared separately in methanol, and further diluted in 50\% methanol in order to prepare working serial dilution at $5,10,20,40,80,150$ and $250 \mathrm{ng} / \mathrm{mL}$ for Nic and at $10,20,40,80,160,300$ and $500 \mathrm{ng} / \mathrm{mL}$ for Cot. For preparation of spiked calibration curve levels, $50 \mu \mathrm{L}$ from these solutions were added to $450 \mu \mathrm{L}$ of Nic-free blood. Similarly, another set of serial dilution were prepared for quality control (QC) samples at $5 \mathrm{ng} / \mathrm{mL}$ for Lower Limit of Quantification (LLOQ), $15 \mathrm{ng}$ / $\mathrm{mL}$ for $\mathrm{QC}_{\text {low }}, 125 \mathrm{ng} / \mathrm{mL}$ for $\mathrm{QC}_{\text {mid }}$ and $200 \mathrm{ng} / \mathrm{mL}$ for $\mathrm{QC}_{\text {high }}$ for Nic and at $10 \mathrm{ng} / \mathrm{mL}$ for LLOQ, $30 \mathrm{ng} / \mathrm{mL}$ for $\mathrm{QC}_{\text {low }}, 250 \mathrm{ng} / \mathrm{mL}$ for $\mathrm{QC}_{\text {mid }}$ and $400 \mathrm{ng} / \mathrm{mL}$ for $\mathrm{QC}_{\text {high }}$ for Cot.

The DBS for standard and QC samples were prepared from freshly spiked whole blood and $60 \mu \mathrm{L}$ were spotted immediately on filter cards. After that, the spots were dried under room temperature for $3 \mathrm{~h}$.

\section{Sample preparation}

A single extraction step was followed for DBS analysis from a standard $6.35 \mathrm{~mm}$ punch. Two punches from each level were placed in a polypropylene micro-centrifuge tube and $100 \mu \mathrm{L}$ of $10 \%(\mathrm{w} / \mathrm{v})$ TCA, containing $20 \mathrm{ng} / \mathrm{mL}$ IS, were added for extraction. The mixture were then vortexed for $1 \mathrm{~min}$ and centrifuged for $10 \mathrm{~min}$ at $16,400 \mathrm{rpm}$ by Eppendorf centrifuge (MiniSpin ${ }^{\circledR}$ Plus, $12 \times 1.5 / 2 \mathrm{~mL}$ ). The supernatant was then transferred into autosampler (AS) micro vial.

\section{Method validations}

The developed DBS-based method was validated following the European Medicines Agency (EMA) and United States FDA guideline, ${ }^{14,15}$ in terms of specificity, carryover, sensitivity, linearity, accuracy, precision, matrix effect, recovery and stability. DBS handling process was following a reported procedure. ${ }^{16,17}$ Hematocrit effects for Cot were studied by Sosnoff and reported previously, ${ }^{10}$ and blood spot volume effect was also studied previously. ${ }^{18,19} \mathrm{~A}$ chromatographic effect (or distribution effect) for the analytes due to a decentralized DBS punching area was studied in the current validation to determine the influence of used filter paper on spread of analytes across the spot. ${ }^{17}$

Linearity, accuracy and precision: Within-run linearity, accuracy and precision were evaluated by running analytical sequence containing six replicates from each of LLOQ, QC low, QC mid and QC high with calibration curve including blank and zero. Betweenrun linearity, accuracy and precision were evaluated by running three sets of within-run sequences in three separate analytical batches within two days. For quantitative data calculations, a built up processing method was used by processing setup- Xcalibur software, where targeted analytes were identified with respect to corresponding accurate mass, calibration levels and related IS. The peaks area of the analytes were then divided over corresponding IS peak area and plotted against specified concentrations to obtain linear regression function using $1 / \mathrm{x}$ weighting factor.

Ion suppression, matrix effect: The ion suppression was checked for DBS by synchronized injection of extracted blank, online through post-column infusion for Nic and Cot standard solution. The ion suppressed negative peak was then compared to the analytes total ion chromatogram (TIC). ${ }^{20}$ Matrix effect test was achieved from six different sources of blood (analytes-free blanks) and expressed in terms of matrix factor (MF) through QC low and QC high samples by calculating the ratio of the peak area in the presence of matrix. This was done by comparing the peak area of blank matrix, spiked after extraction with analyte, to the peak area in absence of matrix (pure solution of the analyte). The IS normalized MF (IS-N MF) was also calculated by dividing the MF of the analyte by the MF of the IS.

Recovery: Nic and Cot extraction recovery from DBS was investigated by applying a second extraction for the reconstituted punches at QC high level, to assure that first reconstitution step has released Nic and Cot in maximum extraction efficiency.

Stability: All stability sections in the validation were performed in triplicate analysis for QC samples at low and high levels, the measurements were then back calculated upon freshly prepared calibration curve. The stock solution and DBS samples were kept under room temperature for $48 \mathrm{~h}$ to check their short term stability and the supernatant stability for the extracted DBS samples at $5^{\circ} \mathrm{C}$ was checked for $48 \mathrm{~h}$. For long term stability another set of QC samples were kept in deep freezer at $-20^{\circ} \mathrm{C}$ for 3 months before analysis. 
Chromatographic effect and decentralized DBS punching area: DBS decentralized punching area could lead to a variable measurement factor, due to the interaction possibility of analyte in blood with the used filter paper materials and through stretching of liquid blood spot over cards. Such interactions could lead to differences in analytes concentration between the center and peripheral areas within a spot. Here, we evaluated the chromatographic effect by punching DBS at peripheral areas (but not touching the spot edge) in six replicate analysis at $\mathrm{QC}_{\text {low }}$ and $\mathrm{QC}_{\text {high }}$ level, and then compared their measurements to corresponding centralized punched samples.

\section{Results and Discussion}

\section{Orbitrap-MS analysis}

Figure 1 shows the detection of protonated molecular ions for Nic, Cot, and Nic-d3 by Orbitrap-MS in H-ESI positive mode, with high mass accuracy, sensitivity and selectivity at $\mathrm{m} / \mathrm{z} 163.12354$, 177.10287 and 166.14235 , respectively.

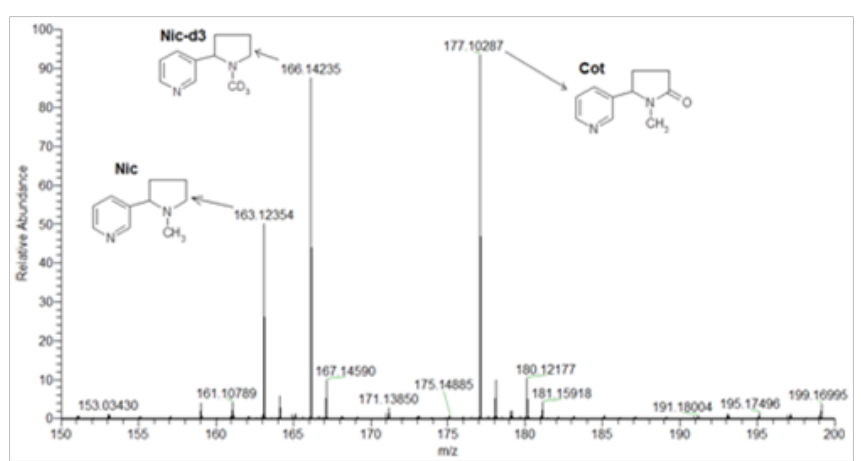

Figure I Orbitrap mass spectrums by ESI positive detection mode for protonated molecular ions $\mathrm{m} / \mathrm{z}$ of Nic, Cot and Nic-d3.
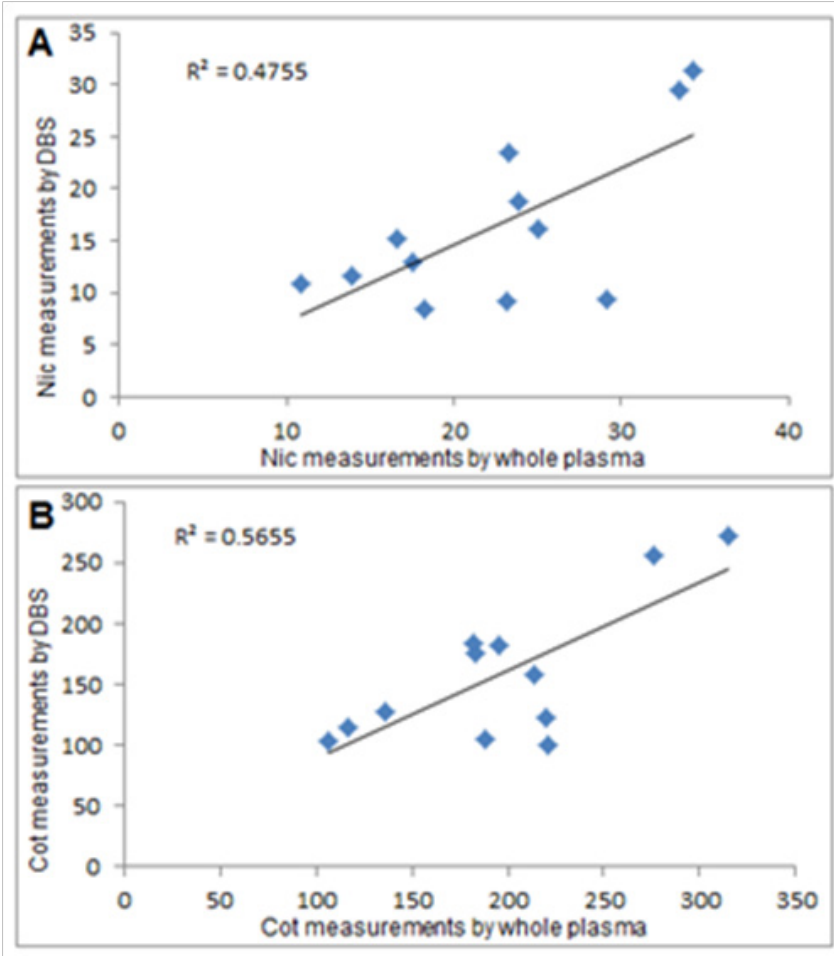

Figure 2 Correlation between DBS measurements with their corresponding measurements from whole plasma for $\mathrm{Nic}(\mathrm{A})$ and $\mathrm{Cot}(\mathrm{B})$.

\section{Chromatographic separation, ion suppression and matrix effect}

The chromatographic conditions for Nic, Cot and IS where optimized for sharp peaks with minimum matrix effects and ion suppression, assuring high throughput by short run time. The retention times for Nic and Cot were 1.8 and 2.0min, respectively, as shown in Figure 3A for LLOQ by DBS. The specificity of extracted DBS for Nic and Cot determination in blood was indicated by analysis the DBS blank from six different blood sources. All blank samples were clean and no endogenous interfering peak were observed from blank screening (Figure 3B) compared to LLOQ (Figure 3A).

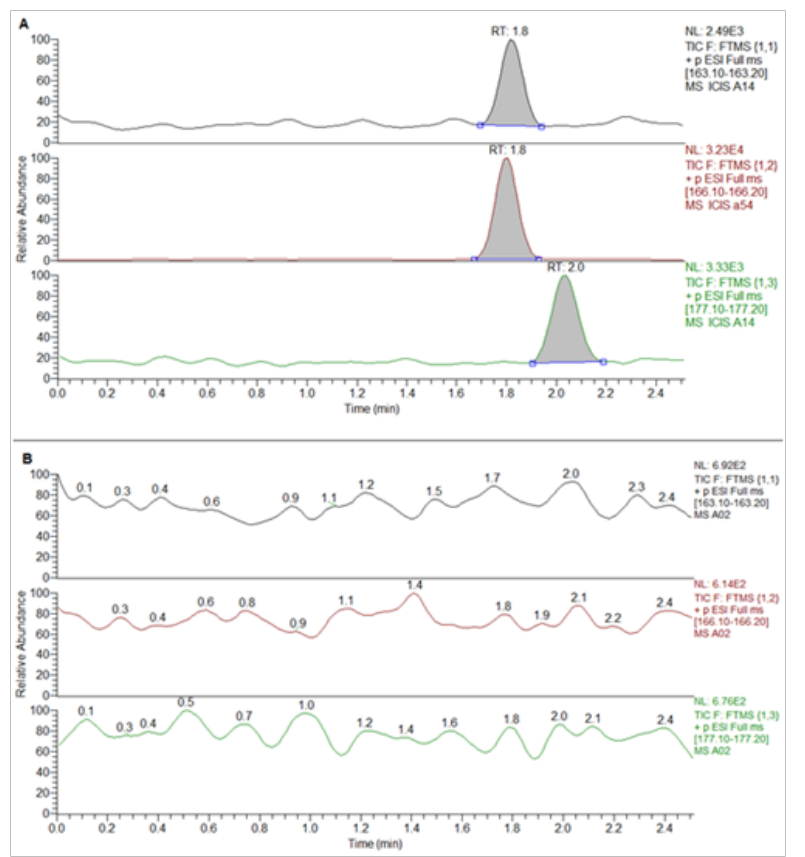

Figure 3 Chromatograms for LLOQ from DBS analysis (A), and extracted blank from DBS (B).

Figure 4 demonstrates that $\mathrm{Nic}$ and Cot peaks in total ion chromatogram TIC, (Figure 4A) are eluted from the column later than ion suppression negative peaks, where the synchronized injection for DBS blank caused a negative peaks through post-column infusion for Nic and Cot solution as shown in Figure 4B \& 4C, respectively. More information about matrix effect was obtained from Table 1 for matrix effect test and reported in terms of MF and IS-N MF for each analyte in blood.

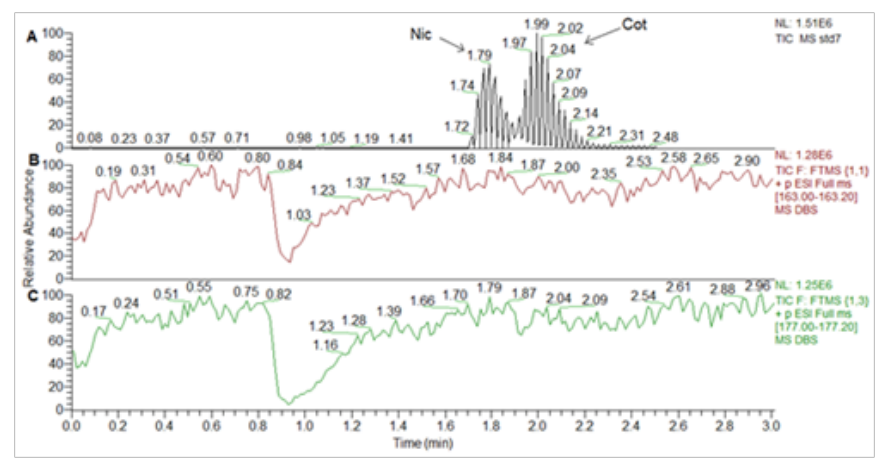

Figure $4 \mathrm{~A}$ comparison TIC chromatogram in A to ionic suppression negative peaks in B and $C$ for Nic and Cot, respectively. 
Table I Matrix factor and internal standard normalized Matrix factor for DBS measurements at QC low and QC high

\begin{tabular}{|c|c|c|c|c|c|c|c|c|}
\hline & \multicolumn{4}{|c|}{ Matrix Factor $(n=6)$} & \multicolumn{4}{|c|}{ Internal Standard Normalized Matrix Factor $(n=6)$} \\
\hline & QC Low & CV \% & QC High & CV \% & QC low & CV \% & QC high & CV\% \\
\hline Nicotine & 1.01 & 1.74 & 0.99 & 2.15 & 1.02 & 2.18 & 0.98 & 1.16 \\
\hline Cotinine & 1 & 1.98 & 0.99 & 2.33 & 1.01 & 3.27 & 0.98 & 1.6 \\
\hline Internal standard & 0.99 & 1.59 & $\mathrm{I}$ & 2.28 & & & & \\
\hline
\end{tabular}

Table 2 DBS measurements accuracy and precision for within-batch and between- batches analytes during validation

Within-Day Measurements (ng/mL, $\mathrm{n}=6$ )

\begin{tabular}{|c|c|c|c|c|c|c|c|c|}
\hline \multirow[t]{2}{*}{ QC Level } & \multicolumn{4}{|c|}{ Nicotine } & \multicolumn{4}{|c|}{ Cotinine } \\
\hline & Spiked & Measured & Accuracy \% & RSD \% & Spiked & Measured & Accuracy \% & RSD \% \\
\hline LLOQ & 5 & 5.27 & $105.39(88-1 \mid 4)$ & 10.36 & 10 & 9.51 & $95.08(8|-| I 5)$ & 12.73 \\
\hline Low & 15 & 15.14 & $100.94(9|-| 08)$ & 5.35 & 30 & 30.28 & $100.93(94-106)$ & 3.99 \\
\hline Mid & 125 & 121.95 & $97.56(93-103)$ & 3.65 & 250 & 246.15 & $98.46(93-103)$ & 3.12 \\
\hline High & 200 & 199.16 & 101.72 (97-107) & 12.34 & 400 & 388.43 & $97.11(95-100)$ & 2.22 \\
\hline \multicolumn{9}{|c|}{ Between-Days Measurements (ng/mL, $n=\mid 8)$} \\
\hline LLOQ & 5 & 5.14 & $102.85(88-117)$ & 8.42 & 10 & 9.74 & $97.36(8 I-I I 5)$ & 8.54 \\
\hline Low & 15 & 14.82 & $98.77(91-108)$ & 4.35 & 30 & 30.95 & $103.16(88-115)$ & 6.46 \\
\hline Mid & 125 & $|24.6|$ & $99.69(93-105)$ & 3.53 & 250 & 247.15 & $98.86(92-114)$ & 4.53 \\
\hline High & 200 & 201.33 & $101.37(97-107)$ & 2.87 & 400 & 399.23 & $97.43(93-102)$ & $2.4 I$ \\
\hline
\end{tabular}

RSD \%: Relative Standard Deviation; LLOQ: Lower Limit of Quantification; QC: Quality Control.

Table 3 Chromatographic effect test by decentralized disk punching for nicotine and cotinine analysis, in terms of measurements accuracy \%

\begin{tabular}{|c|c|c|c|c|c|c|c|c|}
\hline & \multicolumn{4}{|c|}{ Centralized Punching Measurements Accuracy \% } & \multicolumn{4}{|c|}{ Decentralized Punching Measurements Accuracy \% } \\
\hline & \multicolumn{2}{|c|}{ Nicotine } & \multicolumn{2}{|c|}{ Cotinine } & \multicolumn{2}{|c|}{ Nicotine } & \multicolumn{2}{|c|}{ Cotinine } \\
\hline & QC low & QC high & QC low & QC high & QC low & QC high & QC low & QC high \\
\hline Test I & 100.98 & 97.44 & 98.27 & 95.56 & 88.971 & 91.7 & 101.06 & 93.69 \\
\hline Test 2 & 107.65 & 106.32 & 94.54 & 97.67 & 99.319 & 94.54 & 96.83 & 95.43 \\
\hline Test 3 & 101.43 & 104.16 & 101.43 & 99.74 & 102.168 & 94.35 & 91.16 & 99.52 \\
\hline Test 4 & 102.75 & 97.93 & 101.81 & 94.51 & 100.392 & 95.33 & 90.04 & 95.05 \\
\hline Test 5 & 101.71 & 103.75 & 105.94 & 99.37 & 94.849 & 97.06 & 105.24 & 95.57 \\
\hline Test 6 & 91.09 & $100.7 \mid$ & 103.56 & 95.79 & 106.165 & 101.7 & 92.03 & 97.76 \\
\hline Mean & 100.94 & 101.72 & 100.93 & 97.11 & 98.64 & 95.78 & 96.06 & 96.17 \\
\hline RSD \% & 5.35 & 3.54 & 3.99 & 2.22 & 6.09 & 3.53 & 6.35 & 2.18 \\
\hline
\end{tabular}

RSD \%: Relative Standard Deviation; QC: Quality Control

Table 4 Measurements of Nic and Cot in human DBS with corresponding measurements from whole plasma.

\begin{tabular}{lllll}
\hline Subject & \multicolumn{3}{l}{ Measurements by DBS $(\mathbf{n g} / \mathbf{m L})$} & \multicolumn{2}{l}{ Measurements from Whole Plasma $(\mathbf{n g} / \mathbf{m L})^{2 I}$} \\
\hline Nicotine & Cotinine & Nicotine & Cotinine \\
\hline 2 & 10.82 & 103.3 & 10.9 & 106.85 \\
\hline 3 & 9.28 & 123.13 & 23.16 & 220.18 \\
\hline 4 & 8.49 & 105.76 & 18.28 & 188.11 \\
\hline 5 & 12.94 & 182.04 & 17.57 & 195.05 \\
\hline 6 & 31.29 & 271.68 & 34.3 & 314.75 \\
\hline 7 & 16.05 & 157.93 & 25.08 & 213.52 \\
\hline 8 & 11.65 & 114.19 & 13.95 & 117.03 \\
\hline 9 & 9.42 & 100.77 & 29.16 & 220.87 \\
\hline I0 & 15.16 & 175.83 & 16.65 & 183.24 \\
\hline II & 23.51 & 183.21 & 23.24 & 182.29 \\
\hline I2 & 18.87 & 128.31 & 23.89 & 136.3 \\
\hline Mean & 29.54 & 256.74 & 33.51 & 276.49 \\
\hline CV\% & 16.42 & 158.57 & 22.47 & 196.22 \\
\hline t-test & 47.89 & 36.68 & 32.82 & 30.83 \\
\hline
\end{tabular}

DBS: Dried Blood Spot

\section{Standard calibration curve linearity}

The calibration dynamic range for Nic was linear between $5-250 \mathrm{ng} / \mathrm{mL}$. The regression function $(Y=a+b X)$ parameters of $(a=$ intercept, $b=$ slope and correlation coefficient $\left.\left(\mathrm{R}^{2}\right)\right)$ for Nic $(\mathrm{n}=6)$ were $b=0.0058 \pm 0.0001(\operatorname{RSD} \%=2.4), a=-0.0008 \pm 0.0016$ and $\mathrm{R}^{2}=0.9987 \pm 0.0012(\mathrm{RSD} \%=0.12)$. The calibration range for Cot 
was linear between $10-500 \mathrm{ng} / \mathrm{mL}$, and its regression parameters $(\mathrm{n}=$ 6) were $b=0.0041 \pm 0.0002(\operatorname{RSD} \%=4.14), a=-0.0002 \pm 0.0029$ and $\mathrm{R}^{2}=0.9987 \pm 0.0009(\mathrm{RSD} \%=0.09)$.

\section{Within-and between-run sensitivity, accuracy and precision}

Within- and between-run measurements accuracy for Nic and Cot were all higher than $80 \%$ for LLOQ and $85 \%$ for QC levels. Within- and between-run measurements precision for Nic and Cot were all within $15 \%$ as given in Table 2 for precision and accuracy measurements for Nic and Cot in three separate analytical batches from DBS analysis during validation. Each precision batch includes six replicates for LLOQ, $\mathrm{QC}_{\text {low }}, \mathrm{QC}_{\text {mid }}$ and $\mathrm{QC}_{\text {high }}$

\section{Recovery and stability}

The extraction-reconstitution step by TCA solution for the punched DBS disks was efficient enough to recover the analytes from their solid matrix, where the analysis of secondly extracted QC high samples showed that no detectable signals for Nic or Cot were observed, namely, the highest extraction recovery was reached. For short term stability, the test under room temperature and post preparation at $5{ }^{\circ} \mathrm{C}$ show that Nic and Cot were stable within the specified test time, and the calculated accuracies for Nic and Cot were higher than $85 \%$.

\section{Chromatographic effects}

The data obtained from decentralized disk punching (from peripheral areas) within the DBS circle was compared to the data obtained from the centralized punching analysis as given in Table 3. The data indicated that centralized punches had slightly higher analytes intensities than the decentralized punches. The accuracy and precision for decentralized punching measurements were comparable to corresponding centralized punching measurements. Thus, demonstrate that overall chromatographic variation compares a minimal variation factor in Nic and Cot quantitation by DBS, as long as the punched disks stayed within the confines of the DBS (nontouching the edge).

\section{Application}

Early morning blood spots samples were collected from twelve healthy smoking volunteers over filter cards in duplicate from their hand's fingers, following a DBS processing protocol, ${ }^{16,17}$ synchronized with drawing blood samples into heparinized tubes for plasma analysis. ${ }^{21}$ The collected blood spots were left for $3 \mathrm{~h}$ under room temperature for drying, and then kept in deep freezer at $-20^{\circ} \mathrm{C}$ for long term storage till analysis. DBS sampling were synchronized with whole blood collection from each volunteer in a heparinized tube. These blood samples were separated into plasma and kept in deep freezer at $-20^{\circ} \mathrm{C}$ till analysis.

The measurements of Nic and Cot in DBS with corresponding measurements from whole plasma measurements are listed in Table 4. The scattered measurements in subject's no. 2, 3 and 8 as seen from table 4 for DBS measurements compared to corresponding measurements of whole plasma could be attributed to problems with samples preparation. But in spite of these deviations, a good correlation in Nic and Cot measurements between both sampling methods was indication from t-test $(\mathrm{p}<0.05)$ in Table 4 , where $\mathrm{p}$ -value for Nic and Cot were 0.0051 and 0.0098 , respectively.

Long term storage conditions for such volatile compounds (Nic and Cot) could also attributes the noticed few scattered measurements as illustrated in Figure 2 for DBS compared to corresponding whole plasma measurements

\section{Conclusion}

A high throughput, economic and simple bioanalytical method was developed and validated for the quantitative analysis of Nic and Cot in DBS using LC-Orbitrap MS. Single extraction-reconstitution step by TCA solution was successfully applied for DBS extraction. Chromatographic effect was investigated for first time in this study and demonstrated that overall variation account due to the decentralized punching and hematocrit effect comprised a minimal variation factor in Nic and Cot quantitation by DBS as long as the punched disks stayed within the confines of the DBS (non-touching the edge).

\section{Acknowledgments}

None.

\section{Conflicts of interest}

Author declares there are no conflicts of interest.

\section{Funding}

None.

\section{References}

1. Benowitz NL. Nicotine Addiction. $N$ Engl $J$ Med. 2010;362(24):2295-2303

2. Ujváry I. Nicotine and Other Insecticidal Alkaloids. Nicotinoid Insecticides and the Nicotinic Acetylcholine Receptor. 1999. p.29-69.

3. Lunell E, Molander L, Ekberg K, et al. Site of nicotine absorption from a vapour inhaler--comparison with cigarette smoking. Eur J Clin Pharmacol. 2000;55(10):737-741.

4. Li W, Tse FLS. Dried blood spot sampling in combination with LC-MS MS for quantitative analysis of small molecules. Biomed Chromatogr. 2010;24(1):49-65.

5. Murphy SE, Wickham KM, Lindgren BR, et al. Cotinine and trans 3'-hydroxycotinine in dried blood spots as biomarkers of tobacco exposure and nicotine metabolism. $J$ Expo Sci Environ Epidemiol . 2013;23(5):513-518.

6. Spooner N, Lad R, Barfield M. Dried blood spots as a sample collection technique for the determination of pharmacokinetics in clinical studies: Considerations for the validation of a quantitative bioanalytical method. Anal Chem. 2009;81(4):1557-1563.

7. Edelbroek PM, van der Heijden J, Stolk LML. Dried blood spot methods in therapeutic drug monitoring: methods, assays, and pitfalls. Ther Drug Monit . 2009;31(3):327-336.

8. Stove CP, Ingels AS, De Kesel PMM, et al. Dried blood spots in toxicology: from the cradle to the grave? Crit Rev Toxicol. 2012;42(3):230-243.

9. Malhotra S, Kim T, Zager J, et al. Use of an oncolytic virus secreting GM-CSF as combined oncolytic and immunotherapy for treatment of colorectal and hepatic adenocarcinomas. Surgery. 2018;141(4):520-529.

10. Connie SS, John TB. Analysis of cotinine in dried blood spots by LC APCI tandem mass spectrometry. Clinica Chimica Acta. 2008;388(1-2):228-229.

11. Spector LG, Hecht SS, Ognjanovic S, et al. Detection of cotinine in newborn dried blood spots. Cancer Epidemiol Biomarkers Prev. 2007;16(9):1902-1905.

12. Tretzel L, Thomas A, Piper T, et al. Fully automated determination of nicotine and its major metabolites in whole blood by means of a DBS online-SPE LC-HR-MS/MS approach for sports drug testing. J Pharm Biomed Anal. 2016;123:132-140. 
13. Sharma A, Jaiswal S, Shukla M, et al. Dried Blood Spots : Concepts, Present Status and Future Perspectives in Bioanalysis. Drug Testing and Analysis. 2014;6(5):399-414.

14. EMA. Guideline on bioanalytical method validation. European Medicines Agency. 2012. p.1-23.

15. http://www.labcompliance.de/documents/FDA/FDA-Others/ Laboratory/f-507-bioanalytical-4252fnl.pdf

16. Coombs RW, Investigator P, Fiscus S. Processing of Dried Blood Spots Standard Operating Procedure. ACTG. 2012. p.1-20.

17. http://www.medtox.com/Resources/Images/2763.pdf

18. Adam BW, Alexander JR, Smith SJ, et al. Recoveries of phenylalanine from two sets of dried-blood-spot reference materials: Prediction from hematocrit, spot volume, and paper matrix. Clin Chem. 2000;46(1):126-128.
19. Mei JD, Alexander JR, Adam BW, et al. Innovative Non- or MinimallyInvasive Technologies for Monitoring Health and Nutritional Status in Mothers and Young Children Use of Filter Paper for the Collection and Analysis of Human Whole Blood Specimens 1. J Nutr. 2001;131(5):1631-1636.

20. Annesley TM. Ion suppression in mass spectrometry. Clin Chem. 2003;49(7):1041-1044.

21. Abu-awwad A, Arafat T, Schmitz OJ. Simultaneous determination of nicotine, cotinine, and nicotine $\mathrm{N}$-oxide in human plasma , semen, and sperm by LC-Orbitrap MS. Anal Bioanal Chem. 2016;408(23):6473-6481. 